\title{
Tethering designer short double-stranded RNA to mRNA for co-delivery of molecularly-targeted adjuvants and antigens towards cancer vaccination
}

Theofilus A. Tockary ${ }^{1, \#}$, Saed Abbasi ${ }^{1, \#}$, Miki Masai $^{2}$, Naoto Yoshinaga ${ }^{3}$, Shigeto Fukushima ${ }^{1}, K$ Kazunori Kataoka ${ }^{1 *}$, Satoshi Uchida ${ }^{1}$,

$4 *$

\# These authors are equally contributed to this work.

*Correspondence to K.K. (k-kataoka@kawasaki-net.ne.jp), and S.U. (suchida@koto.kpu-m.ac.jp)

${ }^{1}$ Innovation Center of NanoMedicine (iCONM), Kanagawa Institute of Industrial Promotion, 3-25-14 Tonomachi, Kawasaki-ku, Kawasaki, 210-0821, Japan

${ }^{2}$ Department of Research, NanoCarrier Co., Ltd., 3-25-14 Tonomachi, Kawasaki-ku, Kawasaki, 210-0821, Japan

${ }^{3}$ Biomacromolecule Research Team, RIKEN Center for Sustainable Resource Science, Saitama, 351-0198, Japan

${ }^{4}$ Medical Chemistry, Graduate School of Medical Science, Kyoto Prefectural University of Medicine, 1-5 Shimogamohangi-cho, Sakyo-ku, Kyoto 606-0823, Japan 


\section{Abstract}

Immunostimulatory adjuvants play pivotal roles in mRNA cancer vaccines. Herein, mRNA architectonics-based approach was employed to enhance immunostimulatory properties of mRNA, by tethering immunostimulatory short 24-base-pair double-stranded RNA (dsRNA) to mRNA via hybridization, for specific stimulation of retinoic acid-inducible gene-I (RIG-I), an innate immune receptor triggering effective cellular immunity. This approach ensures the co-delivery of mRNA and adjuvants to obtain antigen expression and immune activation in the same antigen presenting cells for effective vaccination, and allows the tuning of immunostimulatory effects by controlling dsRNA number to avoid safety concerns associated with excessive immunostimulation. Thorough in vitro screening provided optimal mRNA structure that induces the secretion of a broad spectrum of proinflammatory molecules and effectively activated mouse and human dendritic cells, with minimal effect on antigen translation efficiency of mRNA. The optimal mRNA formulation improved the potency of mRNA vaccine to induce cytotoxic immune responses after encapsulation into mRNA lipoplex, and exhibited high therapeutic efficiency in lung metastatic model of melanoma using mRNA encoding tumor associated antigen. Furthermore, this system is versatile to potentiate vaccination effect with the use of mRNA polyplex micelle.

\section{Introduction}

Messenger RNA (mRNA) vaccines have excellent potential in cancer immunotherapy, with numerous clinical trials of mRNA-based cancer vaccines in progress $(1,2)$. Advantages of mRNA in cancer vaccination include efficient induction of cellular immune responses via major histocompatibility complex (MHC) class I pathway and flexible designing for targeting neo-antigens just by changing mRNA sequences $(3,4)$. Vigorous efforts have been devoted to develop safe and effective immunostimulatory adjuvants, as well as to maximize antigen expression efficiency from mRNA, for robust immunization in cancer vaccines through the past years $(2,5,6)$. Alongside with the orthodox strategies comprising physical mixing of mRNA and adjuvants $(7,8)$, increasing number of studies attempt to incorporate adjuvancy into mRNA nanoparticles, providing simple vaccine formulations with minimal components (9). This approach allows co-delivery of mRNA and adjuvants, ensuring that immune cells expressing antigen are simultaneously stimulated by adjuvants $(10,11)$. Oftentimes, adjuvancy is incorporated into delivery materials, typically into lipid components of the lipid nanoparticles (LNPs), by simply utilizing intrinsic proinflammatory properties of the materials or rationally designing them to stimulate specific immune signaling (12-14). However, the use of adjuvanted delivery materials has several drawbacks. Such materials should possess dual functionalities of mRNA delivery and immunostimulation, which makes it difficult to regulate these two processes independently. Moreover, the delivery materials dissociated from mRNA after administration potentially induce off-target inflammation in cells and tissues that do not show antigen expression from mRNA. Most mRNA vaccines utilize highly immunostimulating delivery materials, typically with reducing mRNA immunogenicity by chemical modification $(15,16)$. Herein, we proposed a contrary approach that increases mRNA immunostimulatory properties with the use of less immunogenic delivery materials, to independently control the processes of mRNA delivery and immunostimulation and to avoid off-target immunostimulation.

For preparing immunostimulating mRNA, mRNA architectonics-based approach was employed, wherein mRNA was hybridized with immunostimulatory double stranded RNA (dsRNA) side chains, to prepare comb-structured mRNA with dsRNA teeth (Figure 1). This approach minimizes the risk of mRNA and adjuvant dsRNA separating before the uptake by target cells. In addition, rapid degradation of RNA-based adjuvants inside the body helps to avoid safety concerns associated with prolonged immunostimulation. Further notably, the intensity of immunostimulation is easily controlled by tuning the number of dsRNA teeth, without affecting the properties of delivery systems. For cancer immunotherapy, teeth were designed to activate retinoic acid- 
inducible gene-I (RIG-I), an innate immune receptor inducing strong cytotoxic immune responses (17, 18). Importantly, RIG-I requires only short dsRNA with 20 base pairs (bp) at minimum for its activation (19), and hybridization of such short dsRNA only minimally increases total RNA dose for vaccination. Comb-structure was precisely designed to maximize RIG-I stimulation, preserve mRNA translational efficiency, and avoid complementary RNA by-products during in vitro transcription (IVT) (20-22). Thorough in vitro screening allowed us to obtain comb-structured mRNA that activated dendritic cells (DCs) efficiently with minimal influence on antigen translation efficiency. Ultimately, the comb-structured mRNA improved the potential of mRNA vaccine to induce cytotoxic immune responses after encapsulation into low immunogenic mRNA carriers, including lipoplex and polyplex, thereby exhibiting high therapeutic efficiency in cancer vaccination.

\section{Results}

\section{In vitro screening provided highly immunostimulating comb-structured $\mathrm{mRNA}$}

RIG-I prefers dsRNA with 5' triphosphate at blunt end as a substrate $(19,23)$. For preparation of comb-structured mRNA stimulating RIG-I, we designed RNA with 5' triphosphate (5'ppp-RNA) and RNA complementary both to 5'ppp-RNA and mRNA (cRNA) (Figure 1). While IVT without 5' cap analogue is a cost-effective method to prepare RNA with 5' triphosphate, IVT is reported to produce contaminant RNA strand complementary to the intended RNA strand mainly via RNA-templated RNA transcription (2022). dsRNA prepared from intended RNA and its complementary contaminant RNA may influence hybridization process and immunostimulatory property of the RNA. To circumvent this concern, the sequence of 5'ppp-RNA was designed to contain no adenine (A) and several uracils (Us). IVT of 5'ppp-RNA in the absence of A avoids the synthesis of contaminant complementary RNA, which should have several As in its sequence. The risk of complementary RNA synthesis may be further reduced by avoiding self-complementary sequence in 5'ppp-RNA, as RNA hybridization to self-RNA in cis or trans through self-complementary sequence plays a major role in complementary RNA synthesis (24). As 5'ppp-RNA, we prepared 24 nt or 40 nt GU-repeat RNA, which lacks self-complementary sequence, as well as RNA with random sequence from guanine (G), cytosine (C) and U containing self-complementary sequence (Supplementary Table S1). cRNA was chemically synthesized to avoid synthesis of IVT by-products (Supplementary Table S2). The complementary sequence in cRNA to mRNA was controlled to be 17 nt, as translational activity and immunogenicity of mRNA was preserved after hybridization of $17 \mathrm{nt}$ complementary RNA according to our previous study (25). We placed $2 \mathrm{nt}$ or $10 \mathrm{nt}$ gap sequence between cRNA sequence complementary to 5 'ppp-RNA and that complementary to mRNA. Gaussia luciferase $(g L u c)$ mRNA was first used for easy quantification of protein translational activity. Successful hybridization was confirmed by ultracentrifugation, which separates RNA oligonucleotide tooth attached to mRNA from those in free form, with free tooth passing through the filter; in this experiment, Cy5-labeled tooth was undetected in the flow-through, showing that almost all Cy5-tooth was successfully attached to mRNA (Supplementary Figure S1).

The design parameters of immunostimulatory teeth, including the lengths and sequences of dsRNA, and lengths of gap sequence in cRNA between two regions complementary to 5'ppp-RNA and mRNA (Figure 1), were optimized based on immunostimulating property of comb-structured mRNA, after its introduction to DC2.4 cells, a murine DC-derived cell line. Lipofectamine LTX, a commonly used lipid-based reagent, was used for mRNA introduction, as its efficient mRNA introduction capability in vitro allows for high-throughput evaluation of immunostimulatory and translational properties of mRNA. Importantly, not all of the lipid-based systems are highly immunostimulatory (14). Indeed, Lipofectamine LTX alone and that loading mRNA without immunostimulatory tooth showed negligible immunostimulating effect in vitro compared with that induced by combstructured mRNA, as shown below (see Figures 2 and 5), allowing reliable functional evaluation of comb-structured mRNA. 
Immunostimulatory property of mRNA was evaluated by quantifying transcripts of proinflammatory genes, interferon $\beta$ (IFN- $\beta$ ) and interleukin 6 (IL-6), $4 \mathrm{~h}$ after mRNA treatment. Firstly, we optimized dsRNA sequences and lengths in the tooth using combstructured mRNA with one tooth. GU repeat tooth induced enhanced expression of proinflammatory transcripts compared to random sequence (Figure 2a,b), possibly because GU-repeat sequence might avoid the synthesis of complementary RNA strand by-products as described above. Elongation of GU repeat tooth from $24 \mathrm{bp}$ to $40 \mathrm{bp}$ resulted in slight reduction in the level of proinflammatory transcripts. Although the mechanism underlying this result is unknown, longer complementary RNA strands might have more freedom to hybridize with each other in unintended positions, hampering the formation of intended dsRNA with $5^{\prime}$ triphosphate at blunt end. In the optimization of gap RNA lengths in cRNA between two regions complementary to 5'ppp-RNA and mRNA, 24 bp GU repeat tooth with 2 nt gap increased the levels of proinflammatory transcripts compared to that with 10 nt gap (Figure $2 \mathbf{c}, \mathbf{d}$ ). Gap length might affect local environment of dsRNA, which may be important for recognition by innate immune receptors at molecular levels. Interestingly, introduction of $24 \mathrm{nt} \mathrm{GU}$ repeat tooth alone, as well as separate introduction of mRNA and $24 \mathrm{nt}$ GU repeat tooth, failed to induce strong immunostimulation, further suggesting the importance of local environment of dsRNA for stimulating innate immunity (Figure 2e,f). These results led us to use 24 nt GU repeat teeth with 2 nt gap in the following experiments throughout this study. Expression levels of proinflammatory transcripts increased after increasing the number of $24 \mathrm{nt}$ GU repeat teeth with 2 nt gap from 1 to 5 (Figure 2g,h).

\section{Comb-structured mRNA activated RIG-I for immunostimulation}

Potential innate immune receptors recognizing comb-structured mRNA include dsRNA receptors, RIG-I (19), melanoma differentiation-associated gene (MDA)-5 (26), and Toll-like receptor (TLR) 3 (27), and a single strand RNA receptor, TLR7 (28). To assess RIG-I involvement, the influence of RIG-I knockout on inflammatory response after mRNA treatment was evaluated, using a macrophage-derived cell line genetically modified to express Lucia luciferase (ILuc) after proinflammatory stimuli, under the promoter responsive to interferon regulatory factors (RAW-Lucia cells). Without RIG-I knockout, comb-structured mRNA with 1, 3, and 5 teeth showed enhanced 1Luc expression in RAW-Lucia cells compared to untreated control, while 1Luc expression after treatment of mRNA without tooth was comparable with that in untreated control (Figure 3a). In contrast, comb-structured mRNA with 1, 3, and 5 teeth did not increase ILuc expression levels in RIG-I knockout cells, compared to untreated control. Further notably, without 5' triphosphate, a critical motif in dsRNA for RIG-I recognition $(19,23)$, lLuc expression levels were comparable between comb-structured mRNA and untreated control, in RAW-Lucia cells without RIG-I knockout. These results highlight a pivotal role of RIG-I in the immunostimulation induced by comb-structured mRNA. On the contrary, knockout of MDA-5 in RAW Lucia cells showed almost no influence on ILuc expression after treatment with comb-structure mRNA. This result suggests that combstructured mRNA possessing 24 bp dsRNA teeth does not stimulate MDA-5, which requires dsRNA longer than 2 kb for its stimulation (26). Involvement of TLRs was studied using a human embryonic kidney-derived cell line, HEK293 cells, genetically modified to express human TLR3 or TLR7, which are denoted as HEK-TLR3 or HEK-TLR7, respectively, while HEK 293 cells originally exhibited negligible level of TLR expression. HEK293 cells without genetic modification for TLR expression are denoted as HEK-null. These three cell lines were further transformed to express secreted embryonic alkaline phosphatase (SEAP) reporter

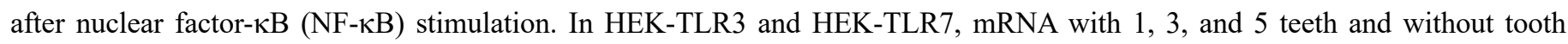
provided SEAP expression levels comparable with those in untreated control, indicating minimal role of TLR3 and 7 in immunostimulation by comb-structured mRNA (Figures 3b). 


\section{Comb-structured mRNA efficiently activated DCs with minimal influence on translational activity}

Detailed functional analyses of comb-structured mRNA were performed using mouse primary bone marrow derived dendritic cells (BMDCs). First, capability of comb-structured mRNA for activating BMDCs was evaluated by measuring expression levels of surface marker proteins, including CD86, CD40, and MHC class I and II (MHC I, and MHC II). After introduction to BMDCs, mRNA with 1 dsRNA tooth significantly increased the expression levels of CD86, CD40, and MHC I compared to mRNA without tooth (Figures 4a-g), demonstrating efficient activation of BMDCs by comb-structured mRNA. The expression level of these markers tended to become maximal at the tooth number of around 3. Meanwhile, effect of dsRNA tooth introduction on MHC II expression was minimal (Figure 4h). Notably, when compared to the treatment with poly I:C, that with mRNA possessing $1-5$ teeth showed enhanced expression of CD86, CD40, and MHC I in BMDCs, demonstrating strong activity of comb structure as an immunostimulatory adjuvant.

The efficiency of antigen expression was studied using gLuc as a reporter. gLuc expression efficiency of combstructured mRNA with 1, 3, and 5 teeth was preserved to approximately $50 \%$ of the efficiency of mRNA without tooth in BMDCs

(Figure 4i). According to previous studies, activation of innate immune responses causes a negative effect on translational activity of mRNA $(29,30)$, which may reduce gLuc expression efficiency after teeth attachment in the present experiment. To study this issue, we introduced $g L u c$ mRNA with 5 teeth without 5' triphosphate, which is non-immunostimulating (Figure 3a). Notably, $g L u c$ expression from mRNA with 5 teeth without $5^{\prime}$ triphosphate was comparable to that from mRNA without tooth. These results indicate that reduction of translational activity after tooth attachment is attributed to the induction of innate immune responses, rather than the process of attaching complementary RNA to mRNA. This is consistent with our previous result that mRNA preserves its translational activity after hybridization with $17 \mathrm{nt}$ complementary RNA (25).

For future clinical translation, the functionalities of comb-structured mRNA were studied also using human DCs. Introduction of 1, 3, and 5 teeth significantly improved the CD86 expression levels compared to mRNA without tooth and poly I:C (Fig. 4j). In a reporter assay using gLuc mRNA, the translational activity of comb-structured mRNA was preserved to approximately half of that of mRNA without tooth (Fig. 4k). These results demonstrate potential utility of comb-structured mRNA in human antigen presenting cells.

\section{Comb-structured mRNA broadly stimulated the expression of proinflammatory molecules}

Further immunological characterization of comb-structured mRNA was performed using multiplex immunoassay and enzymelinked immunosorbent assay (ELISA) to measure protein expression levels of 25 types of cytokines, interferons, and chemokines in total. Twenty-four hours after mRNA introduction to mouse BMDCs, protein concentration of these immune molecules was measured (Supplementary Figure S2), and fold-change compared to untreated control is shown as color gradient in Figure $\mathbf{5}$ for 16 out of the 25 immune molecules, while expression levels of the remaining 9 molecules were below detection limit. mRNA without tooth showed more than 2-fold increase in expression of 2 proinflammatory cytokines (interleukin (IL)-1 $\beta$, IL-6), and 1 chemokine (C-X-C motif chemokine ligand (CXCL)10) compared with that in untreated control and control treated with lipofectamine without mRNA, showing immunostimulatory properties of mRNA. Intriguingly, compared to mRNA without tooth, comb-structured mRNA additionally increased the expression of IL-1 $\beta$ by approximately 2-fold, IL-6 by 17-33-fold, and CXCL-10 by 5-7-fold, in a manner dependent on the number of teeth. Further notably, enhanced secretion of several pro-inflammatory molecules was induced only by comb-structured mRNA, not by mRNA without tooth; Comb-structured mRNA provided approximately 100 -fold increase in the expression levels of a type I interferon (IFN- $\beta$ ), and 2-10-fold increase in that of 2 
proinflammatory cytokines (IL-1 $\alpha$, tumor necrosis factor (TNF)- $\alpha$ ), and 3 chemokines (CXCL1, CC chemokine ligand (CCL)2, CCL5) compared untreated control, whereas mRNA without tooth did not increase the expression of these immune molecules by more than 2-fold. These results show that comb-structure of mRNA strengthens the immunostimulatory property of mRNA and broadens the types of secreted immune molecules. Such potentiation of mRNA immunostimulatory property by comb-structure was not observed in comb-structured mRNA without $5^{\prime}$ triphosphate in teeth, and thus may be attributed mainly to RIG-I-based stimulation of innate immunity. Separate introduction of mRNA and tooth appear to induce relatively high levels of proinflammatory proteins in the heatmap. However, the levels were lower than that observed after introduction of comb-structured mRNA, even when the amount of tooth is equal between these two groups (Supplementary Figure S2), which is consistent with the quantification at mRNA level (Figure 2e,f). Despite the properties of comb-structured mRNA to broadly stimulate the secretion of proinflammatory cytokines, type I interferon and chemokines, comb-structured mRNA provided modest influence on the expression of anti-inflammatory cytokines (IL-10) and T helper 2 (Th2)-related cytokines (IL-4, IL-5, IL-9, IL-13) (31); expression of IL-4, IL-5 and IL-10 was undetected, that of IL-13 was low, and that of IL-9 was unchanged after installation of comb-structure. In this immunological profiling, comb-structured mRNA showed much stronger immunostimulation compared to polyI:C, which is consistent with its enhanced effect of increasing the expression of activation markers on DC surface (Figure 4).

\section{Comb-structured mRNA potentiated lipoplex-based mRNA vaccines against cancer}

Our design concept of mRNA cancer vaccines is to increase adjuvant functionality of mRNA with the use of low immunostimulating delivery carriers (see Introduction for detail). According to a recent report, ionizable lipids played a pivotal role in adjuvancy of lipid-based mRNA carrier, while an mRNA carrier based on a cationic lipid did not show adjuvancy in vaccination (14). Among the systems based on cationic lipids, we selected negatively charged lipoplex similar to that showing promising outcome in clinical trials of cancer vaccines (32). The lipoplex is prepared by mixing cationic liposome and mRNA with excess amount of mRNA in charge ratio, and its intravenous (i.v.) injection resulted in strong protein expression from mRNA in immune tissues such as spleen, thereby providing efficient vaccination effects (33). Interestingly, when encapsulating low immunogenic mRNA with N1-methylpseudouridine modification, this lipoplex exhibited minimal inflammatory responses in the spleen after i.v. injection, and thus contributed to the induction antigen-specific immune tolerance (34). This report showed that lipid components of this lipoplex, as well as modified mRNA, were low immunostimulatory. Lipoplexes loading mRNA without tooth and with 1, 3, and 5 teeth showed average size around $200 \mathrm{~nm}$ with polydispersity index below 0.12 in dynamic light scattering (DLS) measurement (Supplementary

Figure 3a). $\zeta$-potential of these lipoplexes was between $-40--70 \mathrm{mV}$ (Supplementary Figure 3b). Intriguingly, in a reporter assay using comb-structured firefly luciferase ( $f$ Luc) mRNA, mRNA with 1 and 5 teeth exhibited fLuc expression efficiency in the spleen at comparable level with mRNA without tooth at $24 \mathrm{~h}$ after injection (Figure 6a). In flow cytometry analyses, injection of combstructured mRNA with 5 teeth increased the expression of CD86 in splenic CD11c positive DCs compared to that in untreated control and that after injection of mRNA without tooth (Figure $\mathbf{6 b}$ ), demonstrating the potential of comb-structured mRNA for DC activation in vivo.

Vaccination effects were then assessed using mRNA expressing ovalbumin (OVA) model antigen, by in vivo cytotoxicity assay, which quantifies the killing of transplanted syngenic splenic cells presenting OVA antigen in vaccinated mice. Seven days after vaccination, comb-structured mRNA with 1,3, and 5 teeth showed enhanced antigen-specific cytotoxicity compared to mRNA without tooth (Figure 6c). The improved vaccination effect of comb-structured mRNA may be attributed to enhanced activation of DCs in immune tissues (Figure 6b). Enhanced secretion of proinflammatory molecules (Figure 5) may also contribute positively 
to the vaccination effect in comb-structured mRNA. In safety evaluation, we measured serum levels of proinflammatory cytokines. While lipoplexes loading naïve mRNA and comb-structure mRNA with 1 and 5 teeth showed slight increase in the level of IL-6 6 $\mathrm{h}$ after vaccination, the levels returned to normal $24 \mathrm{~h}$ after vaccination (Supplementary Figure S4). Serum levels of IFN- $\beta 6$ and $24 \mathrm{~h}$ after injection of all lipoplexes was below detectable limit. These results demonstrate the safety of comb-structure mRNA.

Then, therapeutic potential of comb-structured mRNA was tested firstly using comb-structure mRNA with 1 tooth, which showed efficient antigen specific cytotoxic effects with minimal safety concerns (Figures 6c, S4). Mouse model was prepared by subcutaneously inoculation of lymphoma cell lines expressing OVA (EG7-OVA). In the prophylactic model, EG7-OVA tumor was inoculated $7 \mathrm{~d}$ after vaccination. Both of comb-structure mRNA and mRNA without tooth suppressed tumor growth more efficiently compared to untreated control (Figure 6d). Although comb-structured mRNA tends to be more effective compared to mRNA without tooth, the difference between these two groups is not statistically significant, due to effective prevention of tumor growth observed in mRNA without tooth, which led us to use more challenging treatment model. In this treatment model, mRNA was injected three times every week, after tumor grew to a certain size, and comb-structure mRNA with 1 tooth induced significant reduction of tumor volume compared to mRNA without comb-structure (Figure 6e).

The therapeutic feasibility of comb-structured mRNA was further assessed using glycoprotein 100 (gp100) antigen, a tumor associated antigen of melanoma, after preparing lung metastasis of B16F0 melanoma cells in mice. Notably, mRNA without tooth encoding gp100 failed to improve survival compared to untreated control (Supplementary Figure S5). In this challenging model, comb-structured mRNA with 5 teeth, as well as that with 1 tooth, was used to obtain maximum vaccination effect. Combstructured mRNA with 1 and 5 teeth significantly prolonged the survival of mice compared to mRNA without tooth, with combstructured mRNA with 5 teeth showing the longest survival (Figure 6f). This result demonstrates the therapeutic potential of combstructured mRNA vaccines in a model closely related to clinical settings.

\section{Comb-structured mRNA was versatile to potentiate polyplex-based $m R N A$ vaccines}

Vaccination effects were also evaluated using polyplex micelle (PM) to evaluate versatility of comb-structured mRNA system. PM is prepared by mixing block copolymer and mRNA in aqueous solution, and possesses a core-shell structure, loading mRNA in the core surrounded by poly(ethylene glycol) (PEG) shell. PM provided efficient mRNA introduction especially after in vivo local delivery (35), with minimal inflammatory responses (36). Thus, we chose two local administration routes: intramuscular (i.m.) and intradermal (i.d) injection. Considering that low inflammatory property of PMs may require strong immunostimulatory functionalities in mRNA in vaccination, we used comb-structured mRNA with 5 teeth for PM preparation, as well as mRNA without tooth for control PM. Both PMs showed average size of $65 \mathrm{~nm}$ with polydispersity index of $0.20-0.23$ in DLS measurement (Supplementary Table S3). In a reporter assay using fLuc mRNA, mRNA without tooth and comb-structured mRNA showed comparable levels of fLuc expression after i.m. and i.d. injection (Figure 7a). In vaccination experiment using $O V A$ mRNA, PMs were injected twice at the interval of 2 weeks, and in vivo cytotoxicity assay was performed 1 week after the second injection. In both administration routes, comb-structure successfully enhanced the vaccination effect for inducing cellular immunity compared to that of mRNA without tooth (Figure 7b). In safety evaluation using blood samples, the levels of IL-6 after i.m. and i.d injection of PM loading comb-structured mRNA with 5 teeth was almost comparable with the background level (Supplementary Figure S6), while IFN- $\beta$ was undetected 6 and $24 \mathrm{~h}$ after these treatments.

\section{Discussion}


In this study, we successfully improved the potential of mRNA vaccine to induce cytotoxic immune responses by mRNA architectonics-based approaches. Comb-structured RNA possessing dsRNA with triphosphate at $5^{\prime}$ blunt end was designed to activate RIG-I, an immune pathway inducing effective cytotoxic immune responses $(17,18)$. According to our mechanistic analyses using RIG-I knockout cells and control comb-structured mRNA possessing dsRNA without 5' triphosphate, an ineffective ligand of RIG-I, comb-structured mRNA specifically activated RIG-I with minimal influence on other innate immune receptors of RNA, including MDA-5, TLR-3, and TLR-7 (Figure 3). Subsequently, DCs were effectively activated in vitro and in vivo (Figures 4, 6b), and secreted various proinflammatory molecules with minimally stimulating the secretion of anti-inflammatory and Th2-related cytokines (Figure 5). mRNA architectonics-based approach of immunostimulation requires only RNA oligonucleotides as an additional component, which is a potential advantage for smooth clinical translation. Remarkably, installation of only one short dsRNA tooth (24 bp) drastically improved the immunostimulatory property of $783 \mathrm{nt} g L u c$ mRNA (Figures 2-5) and potentiated vaccination effect of $1437 \mathrm{nt}$ OVA mRNA and $2027 \mathrm{nt}$ gp100 mRNA (Figures 6, 7), with negligible increase in total RNA doses. In addition, such short dsRNA teeth minimally affected physicochemical properties of mRNA carriers, as shown in Supplementary Figure S3 and Table S3 for lipoplex and PM, respectively.

The approach in the present study has several advantages over our previous system that attempted to increase mRNA immunostimulatory property by hybridizing polyU sequence to polyA (37). Our previous approach, completely relying on IVT for dsRNA preparation, may provide by-product via RNA-templated RNA transcription during IVT, which influences the immunostimulation (20-22). The current approach attempted to avoid such by-product by designing sequences of 5 'ppp-RNA and combining with chemical synthesis of RNA oligonucleotides (cRNA). Furthermore, this approach allows precise designing of blunt dsRNA structure with 5' triphosphate, a preferred substate of RIG-I (19). Notably, in vitro screening revealed the importance of such fine-tuned structural designing, wherein subtle change in the dsRNA structure largely influences the intensity of immunostimulation (Figure 2), although mechanisms underlying such influences remain to be fully elucidated. Interestingly, separate formulation of mRNA and immunostimulatory teeth, as well as single formulation of teeth, provided only modest innate immune responses compared to comb-structured mRNA (Figures 2 and 5). Thus, even if dsRNA teeth were unexpectedly detached from mRNA after administration into the body, the detached teeth would induce minimal off-target inflammation in cells and tissues that do not show antigen expression from mRNA. Lastly, intensity of immunostimulation can be controlled in the present design, by tuning the number of dsRNA teeth introduced onto mRNA strands, which would be important to maximize vaccination effect with minimal safety concerns in future medical applications.

Our system drastically increased the secretion of IFN- $\beta$ in vitro, although the influence of type I interferon on mRNA vaccines is still controversial. Previous studies showed either positive or negative effects of type I interferon on mRNA vaccines, depending on the administration routes and delivery systems $(33,38-40)$. Comb-structure increased cytotoxic immune responses after systemic injection of lipoplex-based system (Figure 6), which is consistent with previous reports showing the positive effect of type I interferon after systemic administration of mRNA lipoplex $(33,38)$. While the type I interferon response may pose safety concern of systemic inflammation, lipoplex loading comb-structured mRNA induced only modest and transient increase in the serum level of inflammatory molecules, IL-6 and IFN- $\beta$ (Supplementary Figure S4). Although type I interferon was reported to negatively affect vaccination after local mRNA delivery $(39,40)$, comb-structured mRNA encapsulated in PM improved the effect on cytotoxic immune response after its local delivery. A mechanistic study indicates the importance of type I interferon expression kinetics on immunological signaling in vaccination, with fast induction of type I interferon posing negative effect on T cell function and delayed induction posing positive effect (40). In this regard, PEG shielding of PMs shows inhibitory effect on the recognition of mRNA by 
innate immune receptors (36), which might delay the expression of type I interferon after injection to the body. Such expression kinetics could explain the positive influence of type I interferon on vaccination after local delivery of PM, although further extensive studies are needed for mechanistically understanding double-edge sword functions of type I interferon, which remains unsettled after long-year researches (29).

Intriguingly, comb-structured mRNA improved vaccination effects of the lipoplex-based mRNA vaccine, with formulation comparable with the lipoplex showing promising outcomes in a clinical trial (33) (Figure 6). Therapeutic potential of comb-structured mRNA was demonstrated in two types of cancer models, subcutaneously inoculated lymphoma expressing OVA, a model neoantigen, and lung metastasis of B16F0 melanoma. Worth notably, our system prolonged the survival of mice in the latter model by targeting tumor-associated self-antigen, which requires to overcome immune tolerance, highlighting outstanding potential of our system in cancer vaccination. Furthermore, the comb-structured mRNA is versatile to potentiate the antigen-specific cytotoxic immune response also in polyplex-based vaccines (Figure 7). Most of the current mRNA cancer vaccines are based on lipids, often utilizing immunogenicity of lipid components (1). By providing additional option of adjuvanting mRNA vaccines with the use of comb-structured mRNA, the present study succeeded in effective vaccination without the use of immunogenic lipid-based system. Without need for incorporating adjuvancy into delivery materials, this approach creates more freedom to focus on other properties of mRNA vaccine delivery carriers, including safety of materials and targeting of antigen presenting cells, thereby broadening future designs of mRNA vaccine.

\section{Materials and Method}

\section{Materials}

$f L u c$ and OVA mRNA were purchased from TriLink $®$ Biotechnologies (San Diego, CA). mRNA encoding gLuc and gp100 was prepared by IVT using mMessage mMachine T7 kit (Thermofisher, Waltham, MA). DNA templates are prepared by inserting protein coding sequences of $g L u c$ (pCMV-GLuc control vector, New England BioLabs, Ipswich, MA) and gp100 (NM_021882) into pSP73 vectors (Promega, Madison, WI) possessing $120 \mathrm{bp}$ polyA/T sequences downstream of a multiple cloning site. cRNA was purchased from Hokkaido System Bioscience (Hokkaido, Japan) (Supplementary Table S2), 5'ppp-RNA was prepared by IVT in the absence of ATP using MEGAshortscript T7 kit (Thermofisher) and DNA template, with sequences listed in Supplementary Table S1. HEPES (4-(2-hydroxyethyl)-1-piperazineethanesulfonic acid) $1 \mathrm{M}$ was purchased from Gibco (Waltham, MA), sodium chloride 5 M was purchased from Thermo Fisher Scientific, while chloroform was purchased from Wako Pure Chemicals Industries (Osaka, Japan). DOTMA (1,2-di-O-octadecenyl-3-trimethylammonium propane (chloride salt)) and DOPE (1,2-Dioleoyl-sn-glycero-3phosphoethanolamine) were purchased from NOF corporation (Tokyo, Japan), while Lipofectamine LTX Plus was purchased from Thermo Fisher Scientific. DC2.4 Mouse Dendritic Cells was purchased from MilliporeSigma (Burlington, MA). RAW-Lucia ${ }^{\mathrm{TM}}$ ISG (wild type), RAW-Lucia ${ }^{\mathrm{TM}}$ ISG-KO-RIG-I, RAW-Lucia ${ }^{\mathrm{TM}}$ ISG-KO-MDA5, HEK-Blue ${ }^{\mathrm{TM}}$ Null, HEK-Blue ${ }^{\mathrm{TM}}$ hTLR3, and HEKBlue ${ }^{\text {TM }}$ TLR7 were purchased from Invivogen (San Diego, CA). E.G7-OVA and B16F0-Luc were obtained from JCRB Cell Bank (Tokyo, Japan). Normal Human Dendritic Cell was purchased from Lonza Group AG (Basel, Switzerland). RPMI-1640 (Fujifilm Wako Chemicals, Osaka, Japan), DMEM (Sigma-Aldrich, St. Louis, MO), GM-3TM Lymphocyte Growth Medium-3 (Lonza), OptiMEM Serum Free medium (Thermofisher), PBS (Fujifilm Wako Chemicals, Osaka, Japan), FBS (Dainippon Sumitomo Pharma Co. Ltd., Osaka, Japan), penicillin-streptomycin (Sigma), normocin (Invivogen), blastidicin (Invivogen), Geneticin selective antibiotic G418 Sulfate (Gibco), granulocyte macrophage colony stimulating factor (GM-CSF) 200-15 (Shenandoah BioTech, Warminster, 
PA). mercaptoethanol (Thermofisher), Glutamax ${ }^{\mathrm{TM}}$ (Gibco), sodium pyruvate $100 \mathrm{mmol} / \mathrm{L}$ (Fujifilm), recombinant human granulocyte-macrophage colony-stimulating factor (GM-CSF) (R\&D Systems, Minneapolis, MN), recombinant human interleukin 4 (IL-4) (R\&D Systems), Alpha MEM with nucleosides (StemCell Technologies, Vancouver, Canada) were purchased for cell culture purposes. PE conjugated anti-Mouse CD40 (eBioScience $\left.{ }^{\mathrm{TM}} 12-0401-81\right)$, CD86 (eBioScience ${ }^{\mathrm{TM}}$ 12-0862-81), MHC Class I (H-2Kb) Monoclonal Antibody (eBioscience ${ }^{\mathrm{TM}}$ 12-5958-82), MHC Class II (I-A) Monoclonal Antibody (NIMR-4) (eBioscience ${ }^{\mathrm{TM}}$ 12-5322-81) were purchased from Thermo Fisher Scientific. TruStain FcX TM anti-mouse CD16/32 antibody (Biolegend 101319), FITC anti-mouse CD86 antibody (Biolegend 105005), APC anti-mouse CD11c antibody (Biolegend 117309), human TruStain $\mathrm{FcX}^{\mathrm{TM}}$ (Biolegend 422301), and PE anti-human CD86 antibody (Biolegend 305405) were purchased from Biolegend (San Diego, CA). Cell staining buffer (BioLegend 420201), Accutase (Sigma-Aldrich), ACK lysing buffer (Thermofisher), CellTrace ${ }^{\mathrm{TM}}$ CFSE Cell Proliferation Kit (Thermofisher), luciferin (Summit pharmaceuticals, Tokyo, Japan), IVT RNA kit, SIINFEKL (Thermofisher), Renilla Luciferase Assay System (Promega) Quanti-Luc (Invivogen), Secreted Embryonic Alkaline Phosphatase (SEAP) (invivogen) were purchased for various cell processing and analysis experiments. IL6 FAM Primer (Applied Biosystem Mm00446190), INFB1 FAM Primer (Applied Biosystem Mm00439552_s1) and Mouse ACTB (Actin, Beta) Endogenous Control (FAM ${ }^{\mathrm{TM}}$ Dye/MGB probe, Non-Primer Limited) (Thermofisher), Applied biosystems Taqman ${ }^{\mathrm{TM}}$ Fast Universal PCR Master Mix (2x) No Amperase ${ }^{\mathrm{TM}}$ (Applied Biosystem) were purchased for PCR experiments. Mouse IL6 Duoset ELISA kit (R\&D systems), and Mouse IFN $\beta$ Duoset ELISA kit (R\&D systems) were purchased for ELISA experiments. C57BL/6J Mice (7 weeks, female) was purchased from Oriental Koubo.

\section{Methods}

\section{Preparation of Comb-structured mRNA}

Comb-structured mRNAs were prepared as HEPES 10mM buffered solution of mRNA, cRNA, and 5'ppp-RNA composed at appropriate molar equivalents. The components were allowed to anneal by heating at $65^{\circ} \mathrm{C}$ for 5 minutes using Takara ${ }^{R}$ PCR Thermal Cycler (Takara Corp., Shiga, Japan), and then gradually cool down to $30^{\circ} \mathrm{C}$ over a time span of 10 minutes before settled down to $4{ }^{\circ} \mathrm{C}$. Hybridization was confirmed by conducting separation analysis to comb-structured mRNA prepared from $O V A$ mRNA, cRNA, and Cy5-labeled 5'ppp-RNA in ultra-0.5 centrifugal filter of MWCO 100K (Merck KgaA, Darstadt, Germany) centrifuged at $4{ }^{\circ} \mathrm{C}$ for 5 minutes at $10,000 \mathrm{G}$. The emission at $670 \mathrm{~nm}$ of the flow through was measured using TECAN plate reader (emission $651 \mathrm{~nm})$.

\section{Preparation of anionic mRNA lipoplex}

Anionic mRNA lipoplex was prepared according to previous report (33). First, a cationic liposome was prepared from DOTMA and DOPE (1:1 molar ratio, $6 \mathrm{mM}$ total lipid concentration) using a thin-film hydration and sonication method. Next, mRNA or combstructured mRNA was dissolved in water containing $\mathrm{NaCl}$ and mixed with the liposome suspension at a nitrogen/phosphate (N/P) ratio of 0.5 , to obtain final $\mathrm{NaCl}$ concentration of $150 \mathrm{mM}$.

\section{Preparation of Lipofectamine LTX with Plus reagent/mRNA lipoplex}

Lipofectamine LTX with Plus reagent/mRNA lipoplex was prepared according to manufacturer's protocol. $3.5 \mu \mathrm{g}$ of mRNA was dissolved in $700 \mu \mathrm{L}$ of Opti-MEM ${ }^{\circledR}$ Reduced Serum Medium containing $3.5 \mu \mathrm{L}$ of Plus ${ }^{\mathrm{TM}}$ reagent. This mRNA solution was then added to a $700 \mu \mathrm{L}$ of Opti-MEM ${ }^{\circledR}$ Reduced Serum Medium containing $8.75 \mu \mathrm{L}$ of lipofectamine ${ }^{\circledR}$ LTX reagent. Lipofectamine LTX 
with Plus reagent/comb-structured mRNA lipoplexes were prepared similarly, with the amount of lipofectamine Plus reagent and lipofectamine adjusted to account for RNA content from cRNA and 5'ppp-RNA.

4. Preparation of mRNA polyplex micelles

A block copolymer composed of $44 \mathrm{kDa}$ PEG and PAsp(DET) [PEG- PAsp(DET)] was synthesized as previously reported (41). Using ${ }^{1} \mathrm{H}$ NMR spectroscopy (400 MHz, JEOL ECS- 400, JEOL, Tokyo, Japan), the polymerization degree of the PAsp(DET) segment was determined to be 59. PEG-PAsp(DET) was dissolved in $10 \mathrm{mM}$ HEPES buffer and mixed with mRNA in the same buffer to obtain polyplex micelles (PM). The final concentration of mRNA or comb-structured mRNA was fixed at $300 \mu \mathrm{g} / \mathrm{mL}$ and $\mathrm{N} / \mathrm{P}$ ratio of 5 .

5. Hydrodynamic diameter $\left(\mathrm{D}_{\mathrm{h}}\right)$ and Zeta potential determination by Zetasizer

DLS was conducted by Zetasizer Nanoseries (Malvern Instruments Ltd., Malvern, UK) to measure $\mathrm{D}_{\mathrm{h}}$ of anionic lipoplexes and polyplex micelles formulation of OVA mRNA with and without teeth. The measurement was performed at a detection angle of $173^{\circ}$ and temperature of $25^{\circ} \mathrm{C}$. Note that for measurement of anionic lipoplex, the refractive index was changed to the refractive index of phospholipid $(\mathrm{RI}=1.45)$. The $\mathrm{D}_{\mathrm{h}}$ was obtained by treating the rate of decay in the photon correlation function by the cumulant method and applying Stokes-Einstein equation. The zeta potential of the structures was determined by laser-doppler electrophoresis using the same machine. According to the obtained electrophoretic mobility, the zeta potential of each sample was calculated according to the Smoluchowski equation: $\xi=4 \pi \eta v e$, where $\eta$ is the viscosity of the solvent, $v$ is the electrophoretic mobility, and e is the dielectric constant of the solvent.

6. Evaluation of pro-inflammatory cytokines of comb-structured mRNA in DC2.4 cells

DC2.4 cells were seeded onto 24 -well plates at the density of $2 \times 10^{5}$ cells/well. $24 \mathrm{~h}$ later, culture medium was replaced with serumfree Opti-MEM medium $(500 \mu \mathrm{L})$, and added with $0.5 \mu \mathrm{g}$ of $g L u c$ mRNA with or without teeth formulated with Lipofectamine LTX and Plus reagents. After $4 \mathrm{~h}$ of the incubation, cellular mRNA was extracted using RNeasy ${ }^{\circledR}$ Mini Kit, converted to complementary DNA (cDNA) using ReverTra Ace ${ }^{\mathrm{TM}}$ qPCR RT Master Mix with gDNA Remover for quantification using real time PCR.

7. Probing of RIG-I and MDA5 involvement to immunogenicity using RAW-Lucia ${ }^{\mathrm{TM}}$ ISG Cells (IFN Reporter Raw 264.7 macrophages)

OVA mRNA (100 ng) with or without teeth formulated with Lipofectamine LTX and Plus reagents in $20 \mu \mathrm{L}$ Opti-MEM medium was added to each well of 96 plates. Thereafter, Raw-Lucia ISG cells (100,000 cells/well) suspended in $180 \mu \mathrm{L}$ of DMEM containing

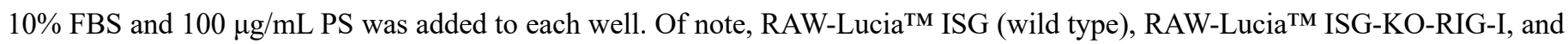

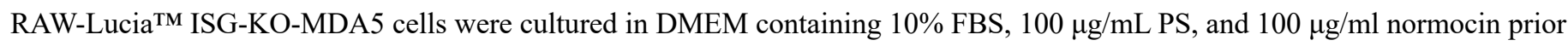
to maintain genetic stability of these transgenic cell lines. After $4 \mathrm{~h}$, the medium was collected for measurement of 1Luc expression was measured.

8. Probing of hTLR3 and TLR7 involvement to immunogenicity using hTLR3-Blue-HEK and TLR7-Blue-HEK293

HEK-Blue ${ }^{\mathrm{TM}}$ Null, HEK-Blue ${ }^{\mathrm{TM}}$ hTLR3 and HEK-Blue ${ }^{\mathrm{TM}}$ TLR7 cells were cultured in selection medium according to manufacturer's protocol. gLuc mRNA (100 ng) with or without teeth formulated with Lipofectamine LTX and Plus reagents in 20 
$\mu \mathrm{L}$ Opti-MEM medium was added to each well of 96 plates. HEK-Blue ${ }^{\mathrm{TM}}$ hTLR3 cells (500,000 cells/well) or HEK-Blue TM TLR7 (400,000 cells/well), and HEK-Blue ${ }^{\mathrm{TM}}$ Null (500,000 cells/well) were suspended in $180 \mu \mathrm{L}$ of SEAP medium were added to each well. After $16 \mathrm{~h}$ of incubation, the plates were examined for absorbance at $630 \mathrm{~nm}$ using TECAN microplate reader.

9. Gene expression, surface antigen presentation and cytokines production in primary BMDCs

Primary BMDCs were obtained according to a previous report (42). In brief, the cells were flushed out from the tibia and femur bone marrow of $\mathrm{C} 57 \mathrm{BL} / 6 \mathrm{~J}$. The cells were cultured at $37^{\circ} \mathrm{C}$ in $5 \% \mathrm{CO}_{2}$ in $\mathrm{R} 10$ culture medium containing (granulocyte macrophage colony stimulating factor (GM-CSF). On day 8, the floating cells in the medium were collected. 400,000 cells suspended in $2 \mathrm{~mL}$ of R10 medium was added to each well of a 12-well plate and transfected on the same day with $1 \mu \mathrm{g} /$ well of gLuc mRNA formulated with Lipofectamine LTX and Plus reagents. $10 \mu \mathrm{L}$ of medium at several time points was collected for gLuc expression assay using Renilla Luciferase Assay System (Promega) and a luminometer, GloMax-Multi+ Detection System (Promega). After 24 h, both floating and attaching cells were collected, washed, blocked with anti-mouse CD16/31 antibody (1/100 dilution in cell staining buffer), and stained with PE-conjugated antibody mouse CD40 (0.005 mg/mL), CD86 (0.005 mg/mL), MHCI (0.007 mg/mL) and MHCII $(0.003 \mathrm{mg} / \mathrm{mL})$ for 20 minutes on ice. The immunostained cells were then washed, stained with DAPI, and examined by flow cytometry Cell Analyzer LSRFortessa X-20 (Becton Dickinson).

In another batch of experiment, the medium from the transfected cells was collected to quantify expression of immune molecules at protein levels. Luminex ${ }^{\circledR}$ Multiplex Assays (ThermoFisher Scientific) was performed to quantify expression levels of 24 molecules, including G-CSF, IFN- $\gamma$, mRNA-1a, 1b, 2, 4, 5, 6, 7, 9, 10, 12p40, 12p70, 13, 15, 17, TNF- $\alpha$, CXCL1, 2, 10, CCL2, 3, 4, 5. Expression of IFN- $\beta$ was quantified using Mouse IFN $\beta$ Duoset ELISA kit.

\section{Human DC}

Human dendritic cell purchased from Lonza was seeded to 12-well plates at 160,000 cells/well containing $2 \mathrm{~mL}$ of medium. Cells were transfected with lipofectamine plus formulation of $m R N A$ mRNA $(0.5 \mu \mathrm{g} / \mathrm{well})$. Adherent and non-adherent cells were harvested after $24 \mathrm{~h}$ for immunostaining. Adherent cells were detached form the plate using $400 \mu \mathrm{L}$ of accutase solution for 5 min and the reaction was stopped using culture medium. All cells were centrifuged at $500 \mathrm{~g}$ for $5 \mathrm{~min}$ at $4^{\circ} \mathrm{C}$. Cells were blocked with $100 \mu \mathrm{L}$ of blocking trustain human antibody (1/250 dilution in cell staining buffer) and kept on ice for 10 min. Then, cells were stained with $100 \mu \mathrm{L}$ of human PE-CD86 solution (BioLegend) (1/40 dilution in cell staining buffer) and kept on ice for 20 minutes. After 2 rounds of washing using $500 \mu \mathrm{L}$ of cell staining buffer, cells were reconstituted with $500 \mu \mathrm{L}$ of staining buffer. The cells were analyzed by flow cytometry using BD LSRFortessa ${ }^{\mathrm{TM}} \mathrm{X}-20$.

\section{Expression of luciferase mRNA in the spleen}

C57BL/6J mice was intravenously injected with $5 \mu \mathrm{g} /$ mouse of $f L u c$ mRNA formulated with anionic lipoplex. After $24 \mathrm{~h}$, spleen was extracted for measuring fLuc expression using IVIS Spectrum SP-BFM-T1 (Perkin Elmer) after intraperitoneal injection of 3 mg of luciferin. fLuc IVIS experiment was also conducted for PMs at $1 \mu \mathrm{g} / \mathrm{mouse}$ for injection via intradermal (i.d.) and intramuscular (i.m) administration. All animal experiments were conducted in accordance with the approval of the animal care and use committees in the Innovation Center of NanoMedicine, Kawasaki Institute of Industrial Promotion (Kanagawa, Japan) and Kyoto Prefectural University of Medicine (Kyoto, Japan). 
12. Examination of surface antigen presentation of dendritic cells in vivo

C57BL/6J mice (7 weeks, female) was intravenously injected with $5 \mu \mathrm{g}$ of OVA mRNA/mouse formulated with anionic lipoplex.

After 24 h, splenocytes were extracted from spleens of donor C57BL6 mice and added with ACK lysing buffer to destroy the red blood cells. $5 \times 10^{6}$ cells were taken and centrifuged at $400 \mathrm{G}$ for $5 \mathrm{~min}$ at $4{ }^{\circ} \mathrm{C}$, and then resuspended in cell staining buffer. The cells were later blocked with $10 \mu \mathrm{g} / \mathrm{mL}$ CD16/32 antibody in $100 \mu \mathrm{L}$ solution and kept on ice for 15 min. Thereafter, the cells were stained with a $100 \mu \mathrm{L}$ mixture of FITC-CD86 $(10 \mu \mathrm{g} / \mathrm{mL})$ and APC-CD11c $(25 \mu \mathrm{g} / \mathrm{mL})$ antibody in cell staining buffer for $20 \mathrm{~min}$ on ice. The cells were washed 3 times and then resuspended in $1 \mathrm{~mL}$ of cell staining buffer for examination using flow cytometry.

\section{In vivo cytotoxic T lymphocyte (CTL) assay}

C57BL/6J mice were vaccinated by intravenous (i.v.) injection of $5 \mu \mathrm{g}$ of $O V A \mathrm{mRNA} / \mathrm{mouse}$ formulated with anionic lipoplex. After 7 days, in vivo CTL assay was performed by following a previous protocol (43). In brief, splenocytes were extracted from donor C57BL6 mice and added with ACK lysing buffer to destroy the red blood cells. The remaining cells were washed and divided into 2 groups. One group was treated with OVA257-264 peptide (SIINFEKL, Toray, Tokyo, Japan) for $1 \mathrm{~h}$ in incubator $\left(37^{\circ} \mathrm{C}, \mathrm{CO}_{2}\right.$ 5\%), and later dyed with $5 \mu \mathrm{M}$ CFSE (CellTrace ${ }^{\mathrm{TM}}$ CFSE Cell Proliferation Kit - For Flow Cytometry, Molecular Probes, Eugene, OR) $\left(1 \mathrm{~mL}\right.$ PBS containing CFSE $5 \mu \mathrm{M}$ per $3 \times 10^{7}$ splenic cells) for $10 \mathrm{~min}$ in water bath $37^{\circ} \mathrm{C}$. The other group was left nontreated, incubated for $1 \mathrm{~h}\left(37^{\circ} \mathrm{C}, \mathrm{CO}_{2} 5 \%\right)$, and later dyed with $0.5 \mu \mathrm{M}$ CFSE $\left(1 \mathrm{~mL}\right.$ PBS containing CFSE $5 \mu \mathrm{M}$ per $3 \times 10^{7}$ splenocytes) for $10 \mathrm{~min}$ in water bath $37^{\circ} \mathrm{C}$. Both groups were concentrated to $5 \times 10^{7}$ splenocytes per $1 \mathrm{~mL}$ PBS, and placed on ice bath before mixed prior to injection into mice. The splenocytes $\left(1 \times 10^{7}\right.$ cells in $200 \mu \mathrm{L}$ of suspension $)$ were intravenously injected to vaccinated mice. $20 \mathrm{~h}$ later, the vaccinated mice were sacrificed to check the viability of the injected CFSE-labelled donor splenocytes by flow cytometry (BD LSRFortessa $\left.{ }^{\mathrm{TM}} \mathrm{X}-20\right)$.

For mRNA-PM vaccines, C57BL6 mice received the vaccine twice at the interval of 2 weeks with $O V A$ mRNA dose set to be 20 $\mu \mathrm{g} /$ mouse for i.m. vaccination, and $12 \mu \mathrm{g} /$ mouse for i.d. vaccination, in each of the prime and boost vaccination. In i.m. vaccination, $20 \mu \mathrm{g}$ mRNA formulated in PM in $100 \mu \mathrm{L}$ solution was divided into two, and injected at the thigh muscle in both left and right sides. In i.d. vaccination, $12 \mu \mathrm{g}$ mRNA formulated in PM in $50 \mu \mathrm{L}$ solution was divided into 5, and injected at 5 locations in the dermis of the back of the mouse. On day 6 post-booster dose, mice were subjected to in vivo cytotoxicity assay as described above.

\section{Prophylaxis tumor suppression study against subcutaneously implanted lymphoma model}

C57BL/6J mice were vaccinated one time with i.v. injection of $5 \mu \mathrm{g}$ of $O V A$ mRNA with or without teeth formulated with anionic lipoplex. After 7 days, mice were subcutaneously implanted with $8 \times 10^{5}$ cells of E.G7-OVA cells. The tumor growth was followed for 2 months. The tumor volume was calculated by the formula $\left(1 / 2 \times \operatorname{long}\right.$ axis $\left.\times(\text { short axis })^{2}\right)$.

\section{Therapeutic tumor suppression study against subcutaneously implanted lymphoma model}

C57BL/6J mice were subcutaneously implanted with $1 \times 10^{6}$ E.G7-OVA cells. Therapeutic intervention was started after 6 days of the inoculation with the tumor growing to average size of $130 \mathrm{~mm}^{3}$ for each group. On this day, and 8 and 15 days later, mice were vaccinated with i.v. injection of $5 \mu \mathrm{g}$ of $O V A$ mRNA with or without teeth formulated with anionic lipoplex. The tumor growth was followed as described in the previous section. 
C57BL/6J mice were intravenously injected with $1 \times 10^{6}$ cells of B16F0-Luc. Therapeutic intervention was started after 6 days. On this day and 5, 9 and 12 days later, $10 \mu \mathrm{g}$ of gp100 mRNA formulated in anionic lipoplex were intravenously injected. Then, the survival of mice was recorded.

17. Safety assay by examining IL-6 and IFN- $\beta$ content in serum

C57BL/6J mice were intravenously injected with $10 \mu \mathrm{g}$ of gp100 mRNA with or without teeth formulated in anionic lipoplex. After 6 and 24 hours, mice were sacrificed to obtain serum. The serum was examined for IL- 6 and IFN- $\beta$ content using ELISA.

\section{Data presentation}

Data are presented as mean \pm standard error of the mean (SEM). In Figures $6(\mathrm{~b}, \mathrm{e})$ and 7 (b) statistical analyses are performed using unpaired two-tailed Student's t test. In Figures 2, 3, 4 (b,c,e,f,g,h,i), 6 (c), and S4 statistical analyses are performed using analysis of variance (ANOVA) followed by Tukey's post hoc test. In Figures $6 \mathrm{f}$ and S5, statistical analyses are performed using log rank test.

\section{Acknowledgements}

This work was supported by Center of Innovation Program (COI) from Japan Science and Technology Agency (JST), Grants-inAid for Challenging Research (Pioneering) [18H05378 to K.K.], for Scientific Research (A) [21H04962 to S.U., 21H04967 to K.K.] and (B) [18K03529 to S.U.] from the Ministry of Education, Culture, Sports, Science and Technology, Japan (MEXT), Leading Advanced Projects for Medical Innovation [21gm0010008s0101 to S.U.], and Research Program on Emerging and Re-emerging Infectious Diseases [21fk0108620h0001 to S.U.] from Japan Agency for Medical Research and Development (AMED). We would like to thank Zheng Wang, Hiroaki Kinoh, Xueying Liu, Joachim Van Guyse, Keisuke Nagao, Hikaru Saitoh, Yuki Sato, Yuki Tada (iCONM), Satomi Nakagahara (NanoCarrier Ltd.), Eger Boonstra (the University of Tokyo) for their technical assistance.

\section{Conflict of Interest}

N.Y., K.K. and S.U. have filed a patent application (Publication No. WO/2018/124181), and NanoCarrier Ltd. (M.M.) holds a right to the patent. K.K. is a Founder and a Member of the Board of NanoCarrier Ltd. M.M. is an employee of NanoCarrier Ltd. .

\section{References}

1. N. Pardi, M. J. Hogan, F. W. Porter, D. Weissman, mRNA vaccines - a new era in vaccinology. Nat. Rev. Drug Discov. 17, 261-279 (2018).

2. L. Miao, Y. Zhang, L. Huang, mRNA vaccine for cancer immunotherapy. Mol. Cancer 20, 41 (2021).

3. U. Sahin et al., Personalized RNA mutanome vaccines mobilize poly-specific therapeutic immunity against cancer. Nature 547, 222-226 (2017).

4. G. Cafri et al., mRNA vaccine-induced neoantigen-specific T cell immunity in patients with gastrointestinal cancer. J. Clin. Invest. 130, 5976-5988 (2020).

5. B. Weide et al., Direct injection of protamine-protected mRNA: results of a phase $1 / 2$ vaccination trial in metastatic melanoma patients. J. Immunother. 32, 498-507 (2009).

6. S. Van Lint et al., Preclinical evaluation of TriMix and antigen mRNA-based antitumor therapy. Cancer Res. 72, 1661- 
1671 (2012).

7. O. A. W. Haabeth et al., mRNA vaccination with charge-altering releasable transporters elicits human T cell responses and cures established tumors in mice. Proceedings of the National Academy of Sciences 115, E9153-E9161 (2018).

8. M. A. Islam et al., Adjuvant-pulsed mRNA vaccine nanoparticle for immunoprophylactic and therapeutic tumor suppression in mice. Biomaterials 266, 120431 (2021).

9. S. Abbasi, S. Uchida, Multifunctional Immunoadjuvants for Use in Minimalist Nucleic Acid Vaccines. Pharmaceutics 13, $644(2021)$.

10. E. Schlosser et al., TLR ligands and antigen need to be coencapsulated into the same biodegradable microsphere for the generation of potent cytotoxic T lymphocyte responses. Vaccine 26, 1626-1637 (2008).

11. N. O. Fischer et al., Colocalized delivery of adjuvant and antigen using nanolipoprotein particles enhances the immune response to recombinant antigens. J. Am. Chem. Soc. 135, 2044-2047 (2013).

12. L. Miao et al., Delivery of mRNA vaccines with heterocyclic lipids increases anti-tumor efficacy by STING-mediated immune cell activation. Nature Biotechnol. 37, 1174-1185 (2019).

13. H. Zhang et al., Delivery of mRNA vaccine with a lipid-like material potentiates antitumor efficacy through Toll-like receptor 4 signaling. Proc. Natl. Acad. Sci. U.S. A. 118, (2021).

14. M. G. Alameh et al., Lipid nanoparticles enhance the efficacy of mRNA and protein subunit vaccines by inducing robust T follicular helper cell and humoral responses. Immunity 54, 2877-2892 e2877 (2021).

15. K. Van der Jeught et al., Dendritic Cell Targeting mRNA Lipopolyplexes Combine Strong Antitumor T-Cell Immunity with Improved Inflammatory Safety. ACS Nano 12, 9815-9829 (2018).

16. Y. Wang, L. Zhang, Z. Xu, L. Miao, L. Huang, mRNA Vaccine with Antigen-Specific Checkpoint Blockade Induces an Enhanced Immune Response against Established Melanoma. Mol. Ther. 26, 420-434 (2018).

17. S. Heidegger et al., RIG-I activating immunostimulatory RNA boosts the efficacy of anticancer vaccines and synergizes with immune checkpoint blockade. EBioMedicine 41, 146-155 (2019).

18. J. Koerner et al., PLGA-particle vaccine carrying TLR3/RIG-I ligand Riboxxim synergizes with immune checkpoint blockade for effective anti-cancer immunotherapy. Nature Commun. 12, 2935 (2021).

19. M. Schlee et al., Recognition of 5' triphosphate by RIG-I helicase requires short blunt double-stranded RNA as contained in panhandle of negative-strand virus. Immunity 31, 25-34 (2009).

20. C. Cazenave, O. C. Uhlenbeck, RNA template-directed RNA synthesis by T7 RNA polymerase. Proc. Natl. Acad. Sci. U. S. A. 91, 6972-6976 (1994).

21. F. J. Triana-Alonso, M. Dabrowski, J. Wadzack, K. H. Nierhaus, Self-coded 3'-extension of run-off transcripts produces aberrant products during in vitro transcription with T7 RNA polymerase. J. Biol. Chem. 270, 6298-6307 (1995).

22. K. Kariko, H. Muramatsu, J. Ludwig, D. Weissman, Generating the optimal mRNA for therapy: HPLC purification eliminates immune activation and improves translation of nucleoside-modified, protein-encoding mRNA. Nucleic Acids Res. 39, e142 (2011).

23. A. Schmidt et al., 5'-triphosphate RNA requires base-paired structures to activate antiviral signaling via RIG-I. Proc. Natl. Acad. Sci. U. S. A. 106, 12067-12072 (2009).

24. Y. Gholamalipour, A. Karunanayake Mudiyanselage, C. T. Martin, 3' end additions by T7 RNA polymerase are RNA selftemplated, distributive and diverse in character-RNA-Seq analyses. Nucleic Acids Res. 46, 9253-9263 (2018). 
25. N. Yoshinaga et al., Induced packaging of mRNA into polyplex micelles by regulated hybridization with a small number of cholesteryl RNA oligonucleotides directed enhanced in vivo transfection. Biomaterials 197, 255-267 (2019).

26. H. Kato et al., Length-dependent recognition of double-stranded ribonucleic acids by retinoic acid-inducible gene-I and melanoma differentiation-associated gene 5. J. Exp. Med. 205, 1601-1610 (2008).

27. I. Botos, L. Liu, Y. Wang, D. M. Segal, D. R. Davies, The toll-like receptor 3:dsRNA signaling complex. Biochim. Biophys. Acta 1789, 667-674 (2009).

28. F. Heil et al., Species-Specific Recognition of Single-Stranded RNA via Toll-like Receptor 7 and 8. Science 303, 15261529 (2004).

29. C. Pollard et al., Type I IFN counteracts the induction of antigen-specific immune responses by lipid-based delivery of mRNA vaccines. Mol. Ther. 21, 251-259 (2013).

30. S. Uchida, K. Kataoka, K. Itaka, Screening of mRNA chemical modification to maximize protein expression with reduced immunogenicity. Pharmaceutics 7, 137-151 (2015).

31. M. P. Kostinov, N. K. Akhmatova, E. A. Khromova, A. M. Kostinova, Cytokine Profile in Human Peripheral Blood Mononuclear Leukocytes Exposed to Immunoadjuvant and Adjuvant-Free Vaccines Against Influenza. Front. Immunol. 11, 1351 (2020).

32. U. Sahin et al., An RNA vaccine drives immunity in checkpoint-inhibitor-treated melanoma. Nature 585, 107-112 (2020).

33. L. M. Kranz et al., Systemic RNA delivery to dendritic cells exploits antiviral defence for cancer immunotherapy. Nature 534, 396-401 (2016).

34. C. Krienke et al., A noninflammatory mRNA vaccine for treatment of experimental autoimmune encephalomyelitis. Science 371, 145-153 (2021).

35. S. Uchida, K. Kataoka, Design concepts of polyplex micelles for in vivo therapeutic delivery of plasmid DNA and messenger RNA. Journal of Biomedical Materials Research Part A 107, 978-990 (2019).

36. S. Uchida et al., In vivo messenger RNA introduction into the central nervous system using polyplex nanomicelle. PLoS One 8, e56220 (2013).

37. S. Uchida et al., Designing immunostimulatory double stranded messenger RNA with maintained translational activity through hybridization with poly A sequences for effective vaccination. Biomaterials 150, 162-170 (2018).

38. K. Broos et al., Particle-mediated Intravenous Delivery of Antigen mRNA Results in Strong Antigen-specific T-cell Responses Despite the Induction of Type I Interferon. Molecular Therapy - Nucleic Acids 5, e326 (2016).

39. V. K. Udhayakumar et al., Arginine-Rich Peptide-Based mRNA Nanocomplexes Efficiently Instigate Cytotoxic T Cell Immunity Dependent on the Amphipathic Organization of the Peptide. Adv Healthc Mater 6, 1601412 (2017).

40. L. Van Hoecke et al., The Opposing Effect of Type I IFN on the T Cell Response by Non-modified mRNA-Lipoplex Vaccines Is Determined by the Route of Administration. Mol Ther Nucleic Acids 22, 373-381 (2020).

41. N. Kanayama et al., A PEG-based biocompatible block catiomer with high buffering capacity for the construction of polyplex micelles showing efficient gene transfer toward primary cells. ChemMedChem 1, 439-444 (2006).

42. M. B. Lutz et al., An advanced culture method for generating large quantities of highly pure dendritic cells from mouse bone marrow. J. Immunol. Methods 223, 77-92 (1999).

43. Y. Yang, C. T. Huang, X. Huang, D. M. Pardoll, Persistent Toll-like receptor signals are required for reversal of regulatory T cell-mediated CD8 tolerance. Nat. Immunol. 5, 508-515 (2004). 


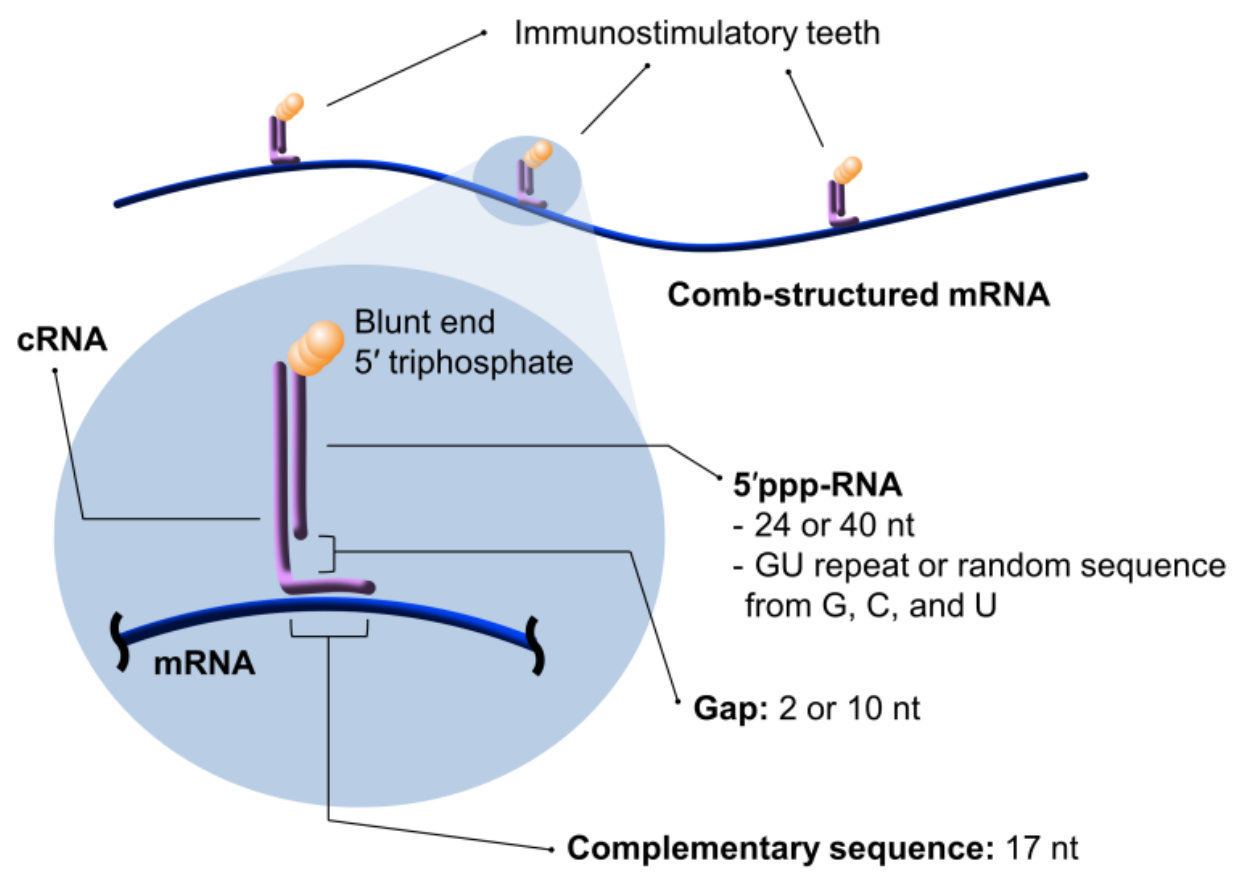

Figure 1. Comb-structured mRNA with immunostimulatory dsRNA teeth. This formulation is prepared from in vitro transcribed RNA with 5' triphosphate (5'ppp-RNA), mRNA, and chemically synthesized RNA complementary both to 5'ppp-RNA and mRNA (cRNA). dsRNA teeth from 5'ppp-RNA and cRNA possess blunt-ended 5' triphosphate, a substrate of RIG-I. Length of cRNA sequence complementary to mRNA is fixed to $17 \mathrm{nt}$ to maintain mRNA translational activity. Design parameters of comb-structured mRNA, including the lengths and sequences of dsRNA teeth, and lengths of gap sequence in cRNA between two regions complementary to 5 'ppp-RNA and mRNA, were optimized. 
(a)

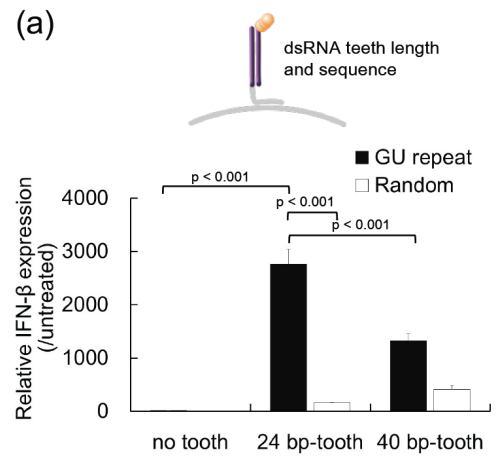

(b)

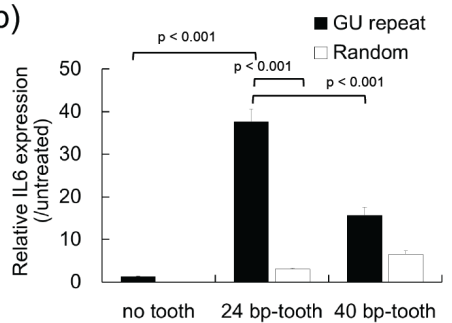

(c)

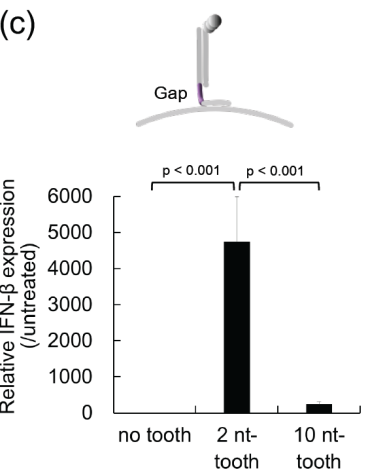

(d)

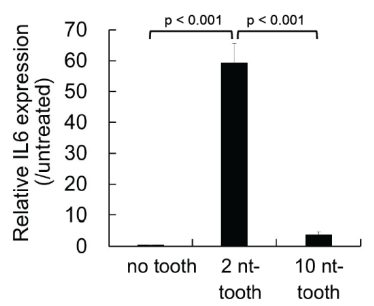

(e)
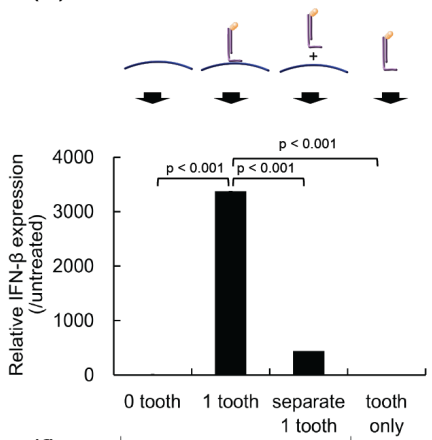

(f)

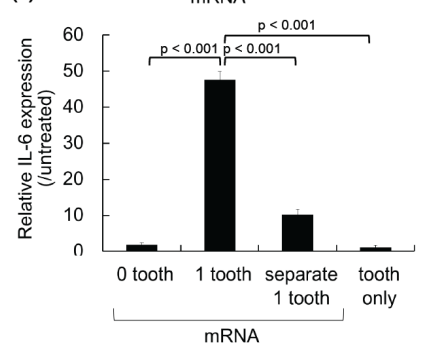

(g)

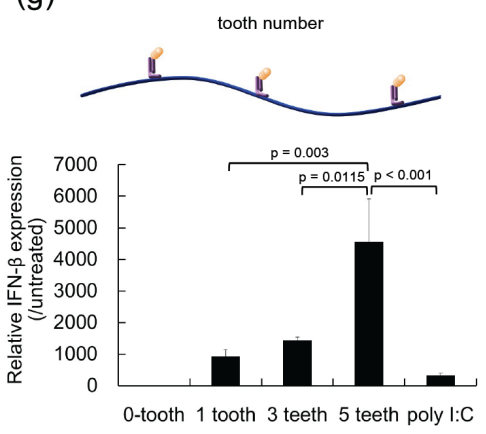

(h)

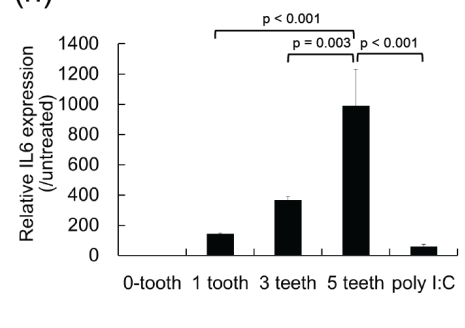

Figure 2. Optimization of tooth design for efficient immunostimulation. Transcript levels of interferon (IFN)- $\beta$ (a, $c, e, g$ ) and interleukin (IL)-6 (b, d, f, h) were measured using quantitative PCR $4 \mathrm{~h}$ after mRNA introduction to DC2.4 cells. (a, b) Effect of dsRNA lengths and sequences. (c, d) Effect of gap RNA lengths in cRNA between two regions complementary to 5'p-RNA and mRNA. (e, f) Effect of dsRNA tooth and mRNA co-delivery method. Introduction of $24 \mathrm{nt}$ GU repeat tooth alone and separate introduction of mRNA and $24 \mathrm{nt} \mathrm{GU}$ repeat tooth were additionally performed. (g, h) Effect of tooth numbers. $n=5$ in a-d, $n=6$ in e-h. 
(a)

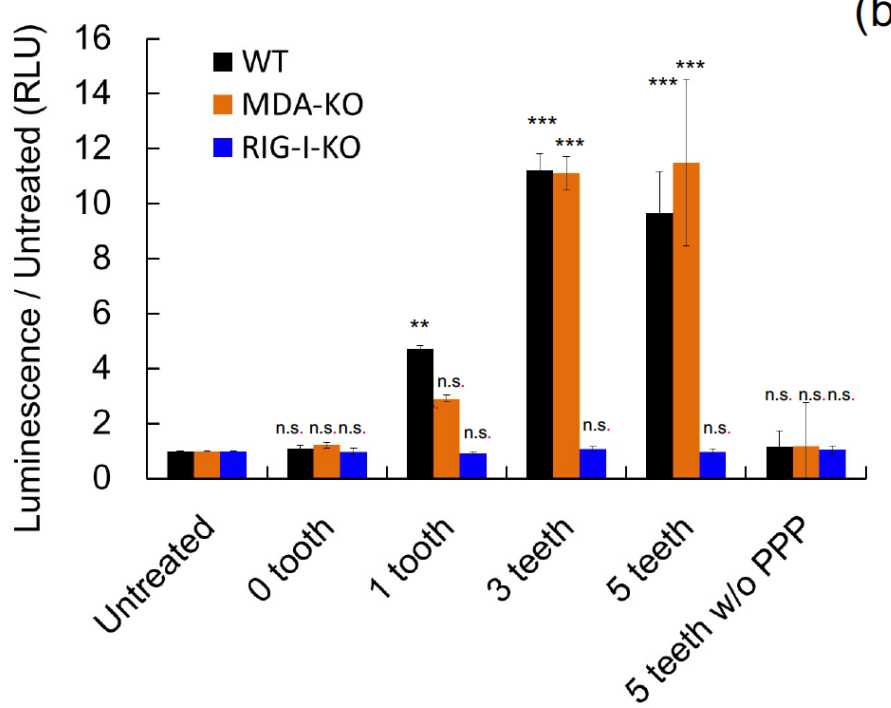

(b)

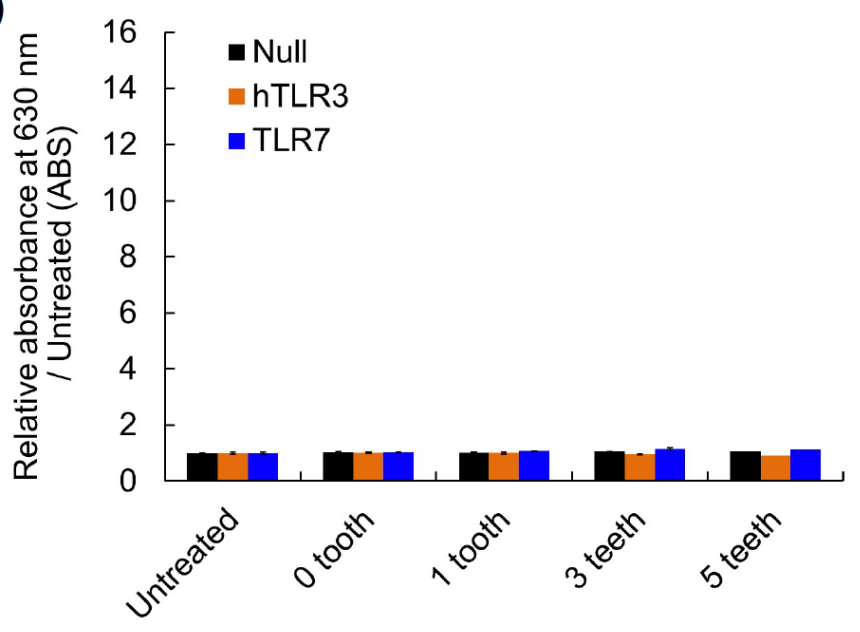

Figure 3. Contribution of innate immune receptors for immunostimulation by mRNA. (a) mRNA was added to RAW cells genetically modified to express Lucia luciferase (ILuc) after proinflammatory stimuli, under the promoter responsive to interferon regulatory factors, with or without knockout of MDA-5 or RIG-I. Reporter 1Luc expression levels represent intensity of immunostimulation. $n=6$. (b) mRNA was added to HEK 293 cells transformed to express secreted embryonic alkaline phosphatase

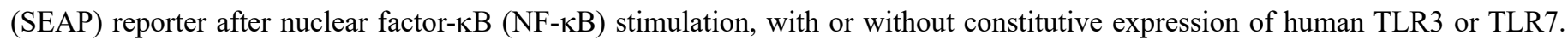
Reporter SEAP expression levels represent intensity of immunostimulation. $n=6$. Abbr.: PPP, $5^{\prime}$ triphosphate. ${ }^{* *}, \mathrm{p}<0.01 ;{ }^{* *}, \mathrm{p}$ $<0.001$, n.s., non-significant versus untreated, respectively. 
(a)

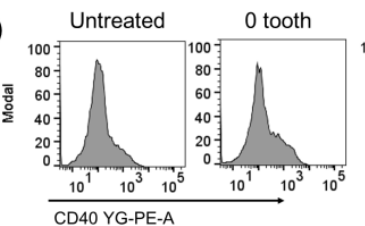

(b)

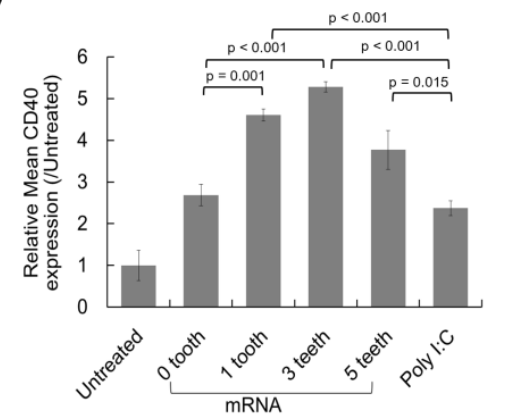

(d)

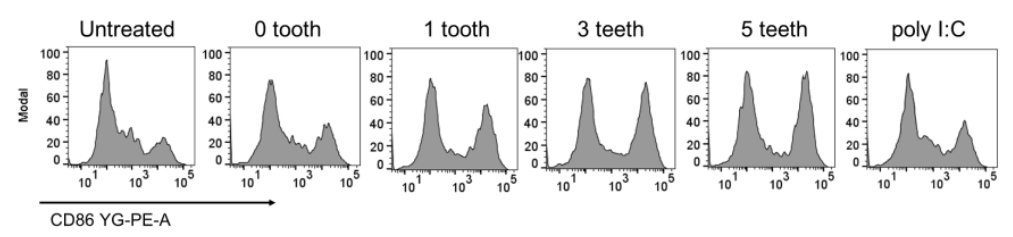

(e)

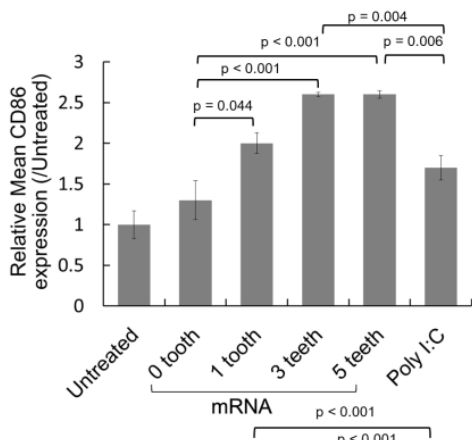

(j)

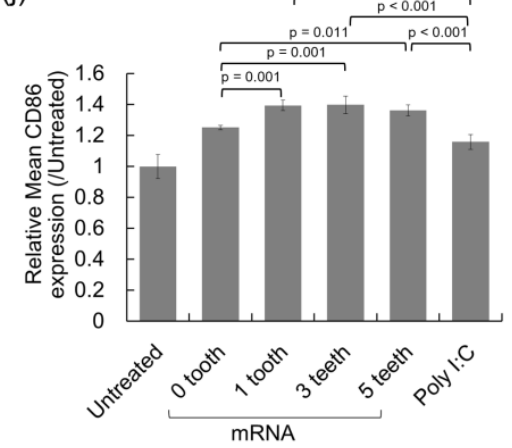

(f)

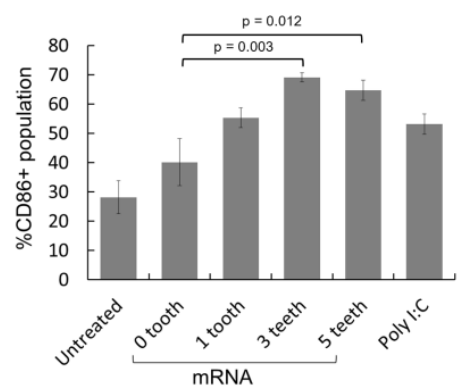

(k) (c)

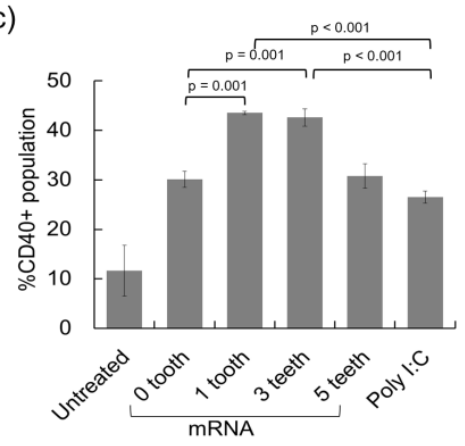

(g)

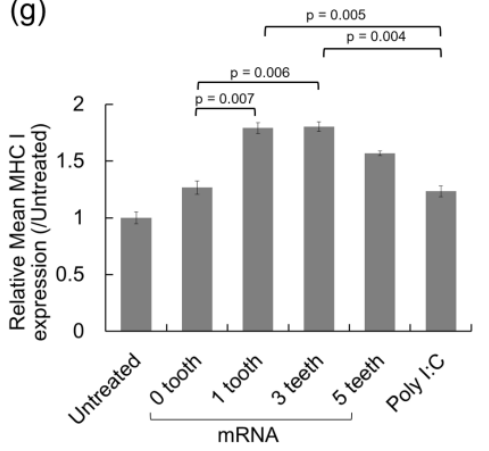

(h)

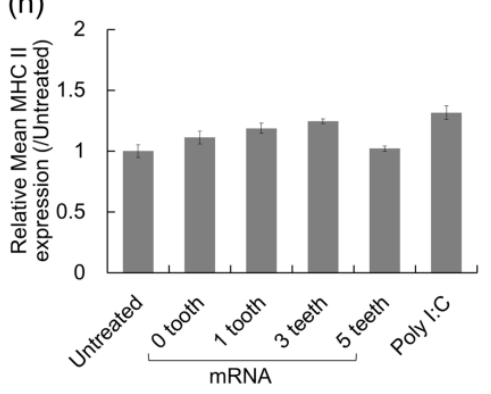

(i)

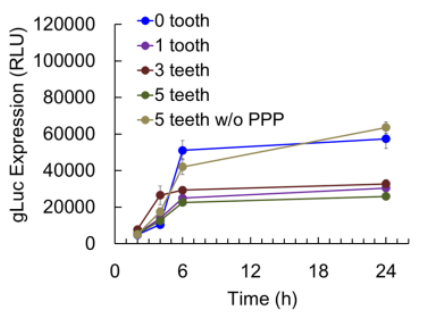

Figure 4. Activation of dendritic cells by comb-structured mRNA. mRNA was added to mouse BMDCs (a-i) and human BMDCs (j, k). (a-h, j) Expression of surface markers, CD40 (a-c), CD86 (d-f, j), MHC I (g), and MHC II (h) was quantified using immunocytochemistry and flow cytometry $24 \mathrm{~h}$ after mRNA addition. $n=4(\mathrm{i}, \mathrm{j})$ Expression of gLuc was measured using cultured medium. $n=6$. 


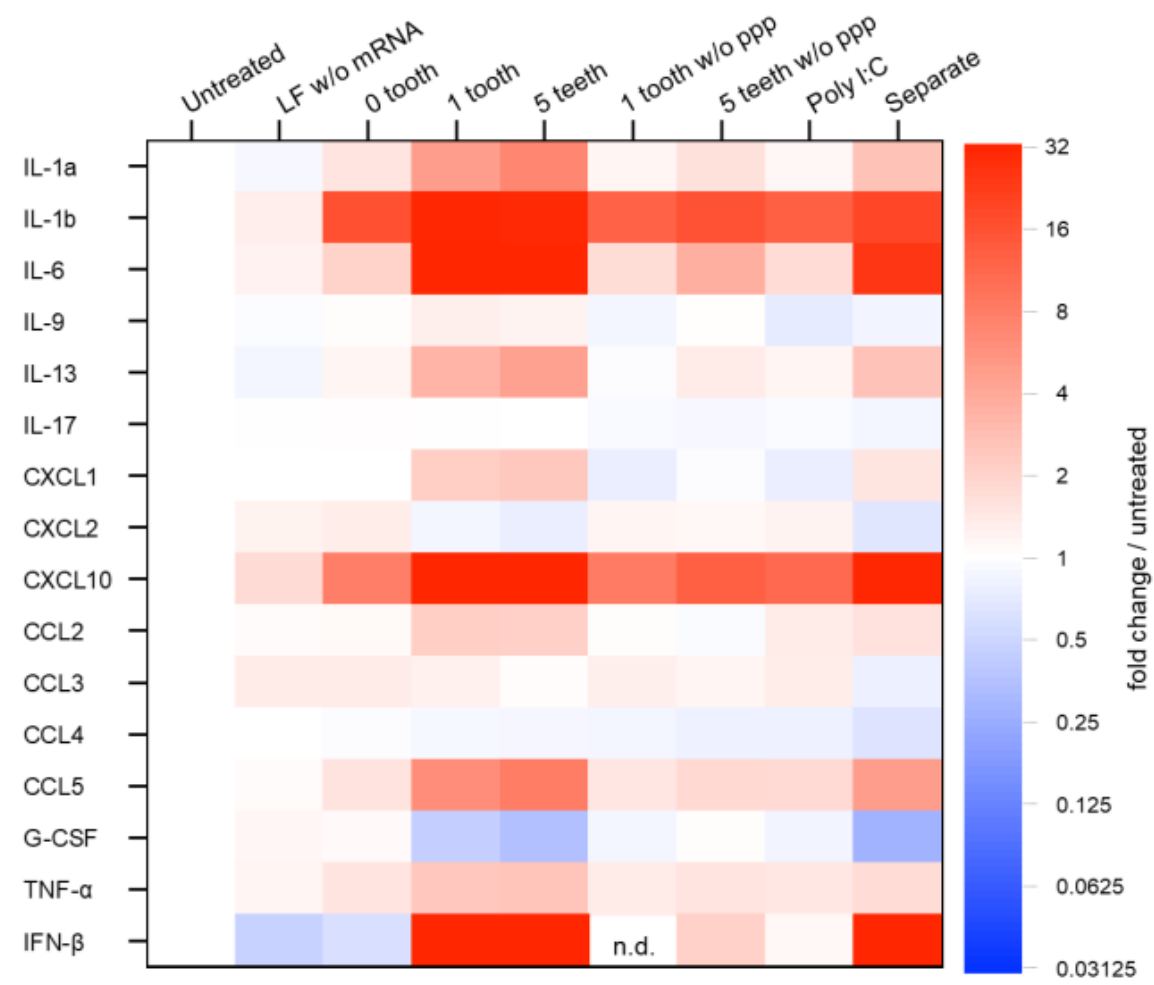

Figure 5. Immunological profiling of BMDCs after mRNA treatment. Protein expression levels of 25 types of cytokines, interferons, and chemokines in total, were quantified $24 \mathrm{~h}$ after mRNA introduction to mouse BMDCs. Colors in the heatmap represent protein levels relative to untreated control. Among 25 types of molecules, 9 types (IFN- $\gamma$, IL-2, 4, 5, 7, 10, 12p40, 12p70, 15) were below detection limits, and data from the other 16 are shown. $n=6$. Abbr.: LF, lipofectamine; PPP, 5' triphosphate; G-CSF, granulocyte colony stimulating factor. In "Separate", mRNA and tooth with tooth amount equal to that in "1 tooth" were separately formulated with LF for addition to BMDCs. 
(a)
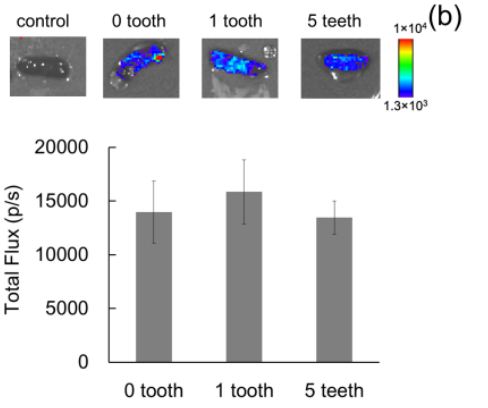

(d)
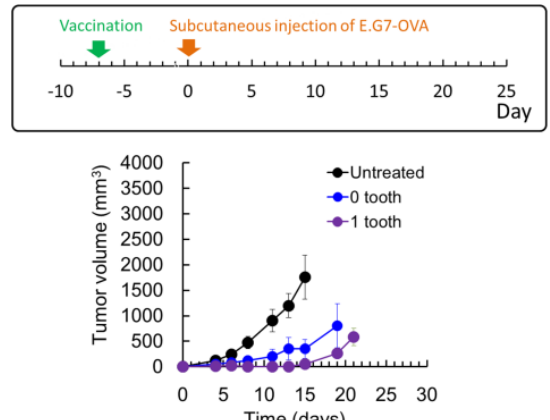

(e)
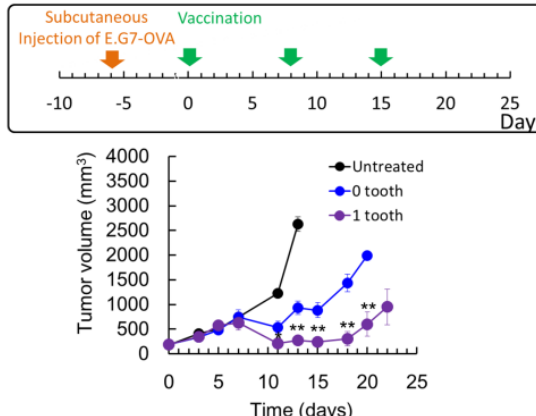

(f)

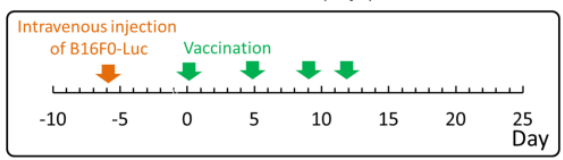

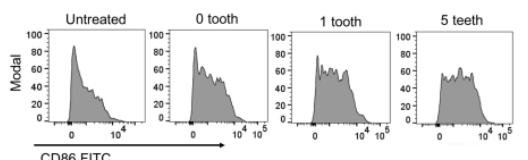
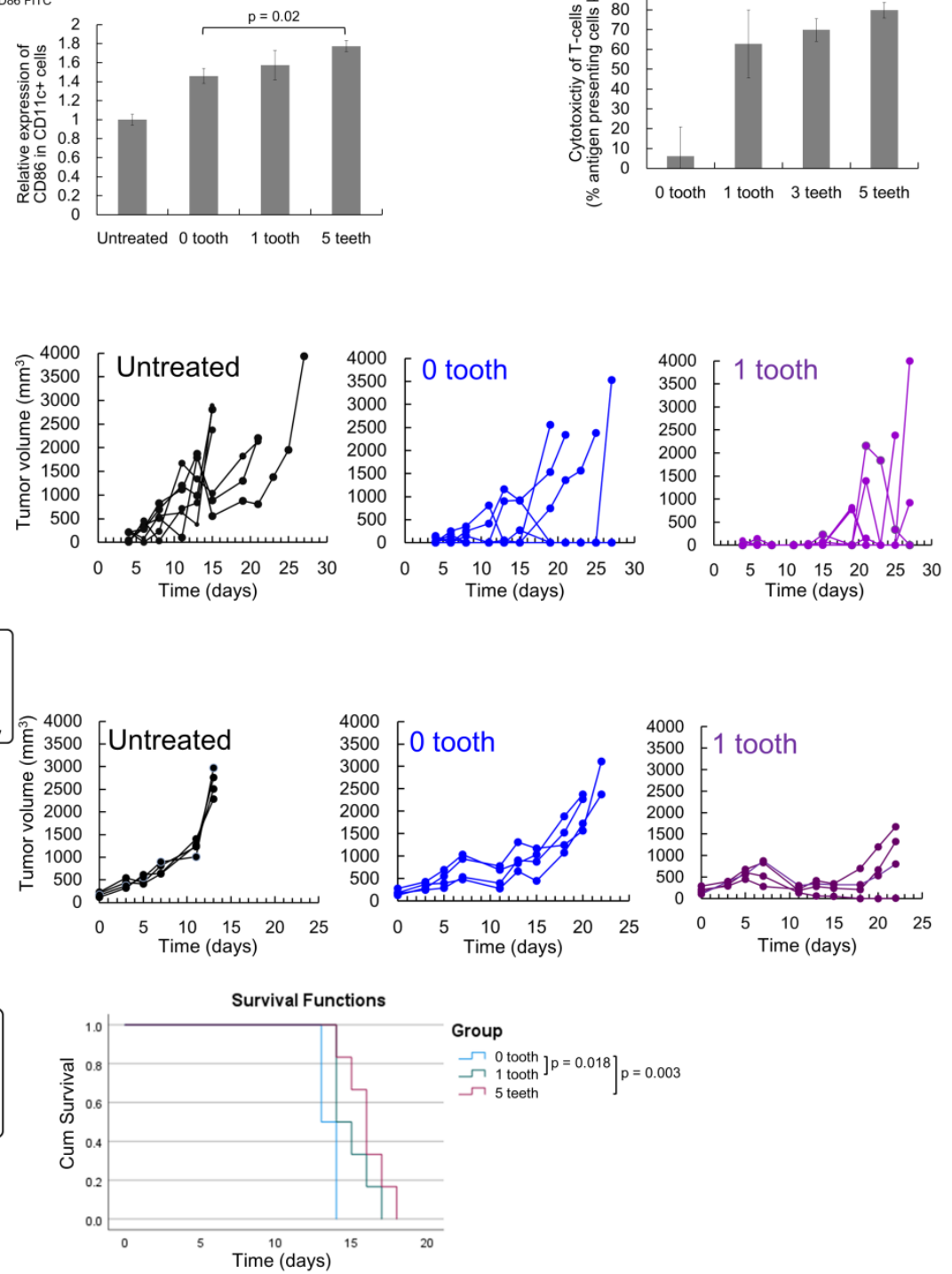

Figure 6. Cancer vaccines using lipoplex in mice. (a) Expression of fLuc in the spleen $24 \mathrm{~h}$ after i.v. injection of lipoplex. $n=4$. (b) Activation of DC in the spleen $24 \mathrm{~h}$ after i.v. injection of lipoplex. $n=4$. Mean expression level of CD86 in CD11c positive splenocytes. (c) Cellular immunity against OVA $7 \mathrm{~d}$ after mRNA vaccination, evaluated by in vivo cytotoxicity assay. $n=4$. (d) Prophylactic model of subcutaneous inoculated lymphoma expressing OVA, treated using OVA mRNA. $n=6$ for untreated, $n=4$ for the other two groups. (e) Therapeutic model of subcutaneous inoculated lymphoma expressing OVA, treated using $O V A$ mRNA. $n=4$. Average of tumor volumes in each group (left) and tumor volume of individual mice are shown in (d, e). (f) Therapeutic model of lung metastasis of melanoma, treated using gpl00 mRNA. $n=6$. 
(a) i.m.

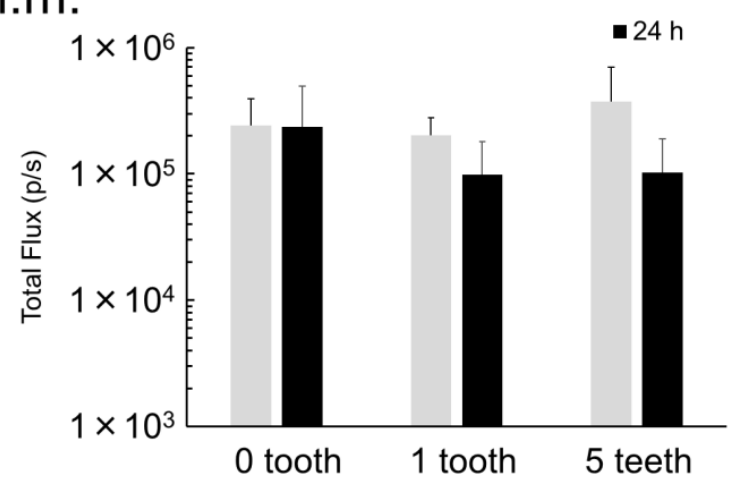

(b)

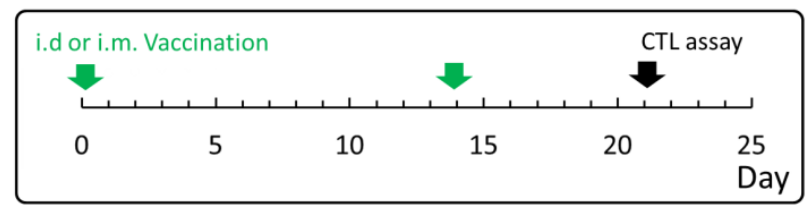

i.d.

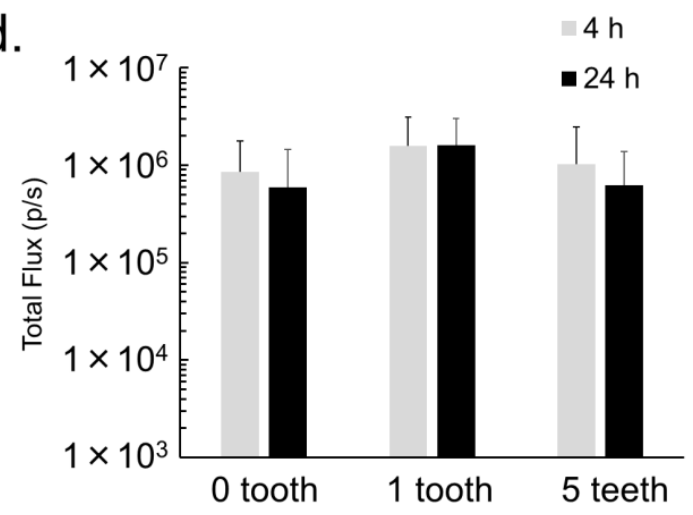

i.m.

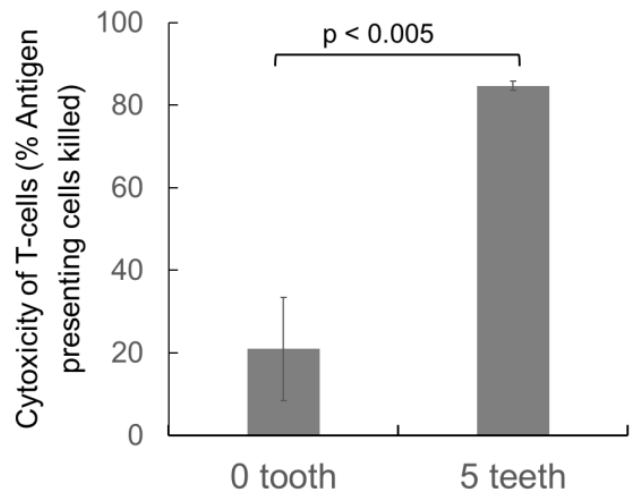

i.d.

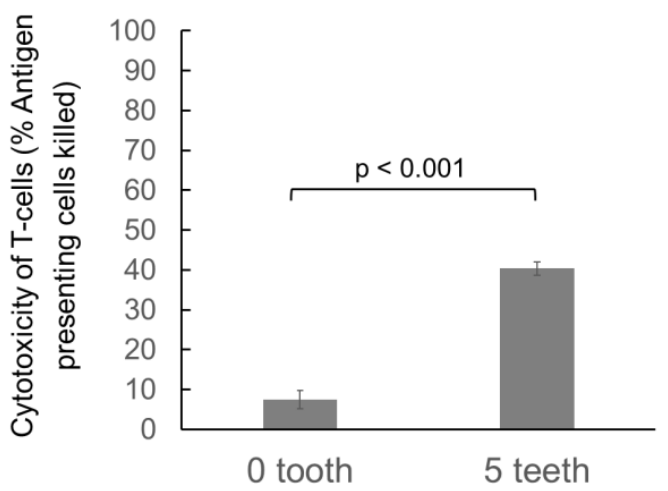

Figure 7. Vaccines using polyplex micelles (PMs) and comb-structured mRNA. PMs loading mRNA without tooth or with 5 teeth were injected to mice via intramuscular (i.m.) and intradermal (i.d.) route. (a) fLuc expression efficiency in the muscle after i.m. injection and in the skin after i.d. injection. $n=3$. (b) In vivo cytotoxicity assay after vaccination using $O V A$ mRNA. $n=4$. 